\title{
Context Aware Power Optimizations of Wireless Embedded Internet Systems
}

\author{
Magnus Lundberg, Jens Eliasson, Linus Svensson, Per Lindgren \\ Luleå University of Technology \\ Department of Computer Science and Electrical Engineering \\ Division of EISLAB \\ SE-971 87 Luleå, Sweden \\ www.eislab.sm.luth.se
}

\begin{abstract}
A key issue in research around embedded Internet systems (EIS) is to reduce power consumption. We envision EIS devices with lifetimes in the range of months or even years. This calls for developing aggressive power management techniques with a high degree of context awareness. As a first step towards this goal we introduce a design methodology for making context aware power optimizations of EIS. The presented design methodology which is verified by experimental results is a promising first step in prolonging operating time of battery powered wireless EIS.
\end{abstract}

\section{INTRODUCTION}

The power consumption of embedded Internet systems (EIS) is an important research issue. Reducing the power consumption is especially important for battery powered devices. Our goal is to prolong the operating lifetime of battery powered EIS devices. To achieve this we need to develop aggressive power management techniques with a high degree of context awareness. As a first step towards this goal we introduce a design methodology for making context aware power optimizations of embedded Internet systems. The proposed approach is to first analyze the components of the system to extract their power/performance characteristics. The next step is to make a trade-off between the application requirements and the power characteristics of the system. After that the context aware power optimizations are implemented and the results are verified through experimental measurements.

To demonstrate this methodology we apply it to a typical embedded Internet system. The system consists of a microcontroller, a generic interface used to attach sensors and a Bluetooth module for wireless communication.

The generic interface allows for a wide variety of sensors to be attached to the platform. Some examples of sensors that are used with this platform are in the area of mobile medical applications and in sporting events, which includes pulse oximeters and accelerometers [1][2]. Pulse oximeters are used to measure pulse rate and blood oxygen saturation and accelerometers are used for monitoring movement of the person under care.

The Bluetooth module makes it possible to communicate with the sensor system either locally using a handheld computer or remotely by routing the traffic onto Internet via a Bluetooth equipped cell phone or a Bluetooth access point. The platform features a lightweight TCP/IP stack and a web server. This enables the data from the sensor to be viewed using a standard web browser.

The outline of this paper is to first describe the system used as an example, this is done in section II. In section III the system is characterized to find possible power optimizations. Section IV discusses the power/performance trade-offs involved in making power optimizations. Section $\mathrm{V}$ describes the experimental setup and the results are discussed in section VI. Section VII holds the conclusion and section VIII describes future work.

\section{SYSTEM DESCRIPTION}

\section{A. Hardware Platform}

The wireless EIS sensor platform is representative for a wide range of wireless embedded system - An IO-interface for interaction with the surroundings, a microcontroller for data processing, a unit for wireless communication and batteries as power supply.

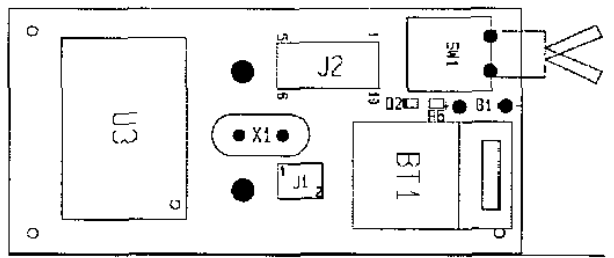

Fig. 1. Wireless EIS platform $(57 \times 27 \mathrm{~mm})$.

For interaction with its surroundings, the EIS platform uses an RS232 serial interface, component $\mathrm{J} 2$ in Figure 1. The interface makes it possible to send and receive data or control signals both to and from a connected sensor. Since RS232 is a widespread standard it is possible to attach a wide range of both custom made and commercially available sensors.

The microcontroller used on the platform is a 16-bit lowpower single-chip component manufactured by Mitsubishi/Renesas (component U3 in Figure 1) [3]. It features $256 \mathrm{kB}$ FLASH and $20 \mathrm{kB}$ RAM as on-chip memories. It requires a supply voltage of $2.2-3.6 \mathrm{~V}$ depending on configuration and clock frequency. In our 
system a $4.608 \mathrm{MHz}$ crystal oscillator (component $\mathrm{Xl}$ in Figure 1) is used as external clock source, even though the microcontroller has capacity to be clocked up to $10 \mathrm{MHz}$. A second $32.768 \mathrm{kHz}$ oscillator is used as sub-clock source that can be used when the microcontroller is operating in lowpower mode. The microcontroller has several other features such as A-D and D-A converters, programmable IO ports and a CRC (Cyclic Redundancy Check) calculation circuit.

The means for wireless communication is Bluetooth, a standardized radio technology suitable for portable devices. The Bluetooth module used is a Mitsumi Bluetooth singlechip class 2 unit with integrated antenna (component BT1 in Figure 1) [4]. It has a nominal range of up to $10 \mathrm{~m}($ at $0 \mathrm{dBm})$ and requires a power supply of $1.8 / 3.3 \mathrm{~V}$. The module is compliant with Bluetooth version 1.1 which, among other things, implies that it has support for different power save modes [5]. Besides the obvious HCI interface to the Bluetooth software stack, parts of the upper layer of the software stack can be run on-chip in the Bluetooth module. More specifically this means that the L2CAP-, SDP- and RFCOMM-protocols can be provided by the module, thus reducing the load on the microcontroller[figure 2]. Finally, the Bluetooth module has a number of external interfaces, including UART- and USB-interfaces.

Requirements such as small size and low weight are often in conflict with requirements of long battery lifetime, leaving the choice of type and size of batteries an open issue. In our experiments the EIS sensor platform was supplied by two rechargeable AAA $1.2 \mathrm{~V} \mathrm{Ni-MH}$ batteries in series with a total capacity of $600 \mathrm{mAh}$. A DC-DC converter is used to step-up the voltage to supply the microcontroller, while the Bluetooth module has a built-in voltage regulator.

\section{B. Software Architecture}

The software required in the sensor platform is naturally application dependent, but by complying with the principle of IP all the way, the need for proprietary protocols can be reduced to a minimum.

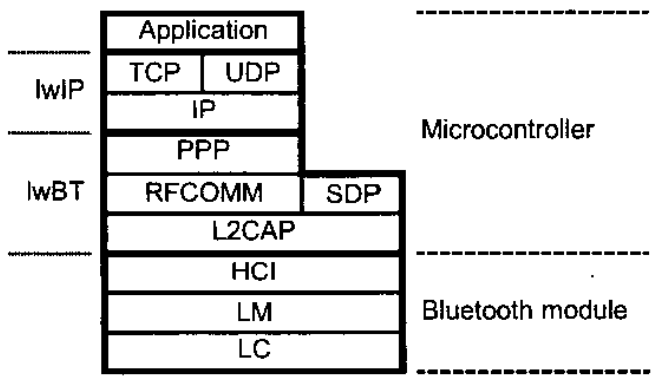

Fig. 2. Software architecture.

The software architecture of the EIS platform is shown in Figure 2. The protocols handled by the Bluetooth module together with the protocols in the lightweight Bluetooth stack
(lwBT) and the lightweight TCP/IP stack (lwIP) makes it possible to communicate with the application using a standard web browser [6][7]. As a result, when new types of sensors are attached to the EIS platform, only the application software needs to be updated.

Both lwBT and lwIP are small independent implementations of the Bluetooth and TCP/IP protocol suites, respectively, with focus on low resource utilization and at the same time providing full functionality.

\section{SYSTEM CHARACTERIZATION}

\section{A. The Bluetooth Module}

The Bluetooth standard specifies, besides active mode, three special modes: Sniff, Hold and Park that can be used to save power. When one of the special modes is used the module is powered down for certain intervals. Instead of using the active mode considerable power savings can be done. The power consumption is specified to be up to 108 $\mathrm{mW}$ in active mode. No power consumption values were specified for the Bluetooth module for the power save modes. But when we measured power consumption for the module in Idle-mode it was $9 \mathrm{~mW}$. This shows that there are considerable power savings to be done using the Sniff mode.

Sniff Mode: When Sniff mode is being used the Bluetooth module is powered down and only wakes up periodically to send or receive data. The number of timeslots the module sleeps can be set between 1 and 65535, where each timeslot is $0.625 \mathrm{~ms}$. This sets the sleep time to a value between 0.625 $\mathrm{ms}$ and $40.9 \mathrm{~s}$.

Hold Mode: Hold mode is very similar to Sniff mode, but Hold mode is non-periodic. The module sleeps for $0.625 \mathrm{~ms}-$ $40.9 \mathrm{~s}$ and then wakes up. While sleeping the module keeps the AM (Active Member) address it was assigned.

Park Mode: Same as Hold mode but the device looses its AM address.

The sleep interval can be set dynamically which makes it possible to adaptively change the sleep interval as the systems context changes. Another option to make the Bluetooth module more power efficient is power control. Power control is used to adjust the signal strength of the radio transmission.

\section{B. Characterization of the Bluetooth Module}

- To characterize the Bluetooth module some measurements where made. The measurements using Sniff mode shows that in some cases significant power savings can be made. The characterization was made with data transmitted using TCP/IP as that was the chosen protocol suite for the sensor system; this was done to get a representative result. 


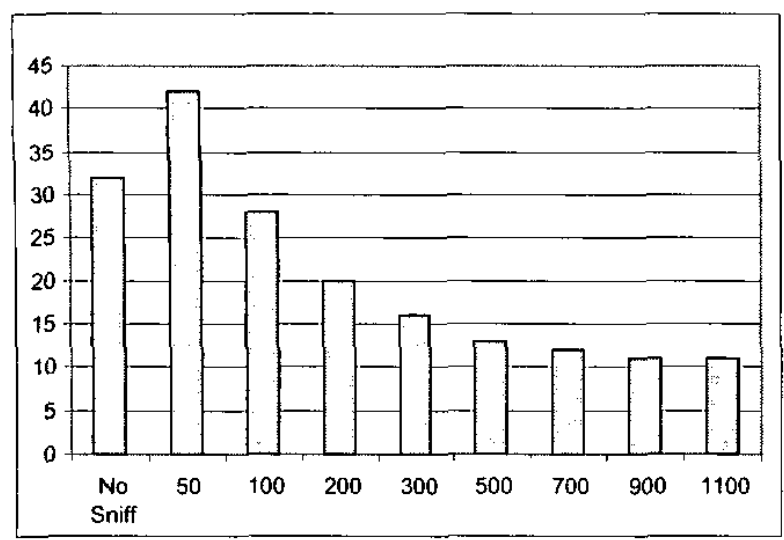

Fig. 3. Power consumption depending on the number of timeslots the modulc sleeps in Sniff mode.

The measurement results in figure 3 shows that allowing the module to sleep for less than 100 timeslots actually increase the power consumption compared to not using Sniff mode. And that if the module sleeps for more than 700 timeslots the power consumption levels out towards $10 \mathrm{~mW}$. Using sleep interval longer of 2000 or more will result in a PPP time out. No characterization of transmission power control was made.

\section{The Microcontroller}

\section{Wait Modes}

The microcontroller has three operating modes: Normal Operation Mode, Wait Mode and Stop Mode [8]. The two later can be used to save power. Instead of allowing the micro-controller to run at full speed when no useful instructions are being performed, the microcontroller can be powered down.

Normal Operation Mode: The microcontroller operates with a system clock, generated by either the main clock or the sub-clock. The internal peripherals operate with individually configured clocks.

Wait Mode: The microcontroller core stops its operation in this mode but oscillators are not affected. This mode is initiated by the WAIT instruction and the microcontroller returns to the normal operation mode upon receipt of an external interrupt request or a hardware reset.

Stop Mode: All oscillators stop in this mode. As a result, not only the microcontroller core but also all internal peripherals stop operating. The microcontroller returns to the normal operation mode upon receiving an external interrupt request or a hardware reset. Resuming from Stop mode can take up to three seconds.

\section{Characterization of the Microcontroller}

In table 1 below the characterization measurements for the microcontroller are shown. The value represents the microcontroller power consumption in Normal, Wait and Stop mode.

Table 2. Power consumption depending on microcontroller operating mode.

\begin{tabular}{|c|c|c|}
\hline Normal Mode & Wait Mode & Stop Mode \\
\hline $48 \mathrm{~mW}$ & $30 \mathrm{~mW}$ & $23 \mathrm{~mW}$ \\
\hline
\end{tabular}

Frequency Reduction

The dynamic power consumption of digital circuits scale linearly with the frequency as shown in equation ( 1 ) where $P$ is the dissipated power, $\mathrm{c}$ is the switched capacitance, $\mathrm{V}$ is the voltage and $f$ is the switching frequency.

$$
\mathrm{P}=\mathrm{cfV}^{2}
$$

Originally the microcontroller was clocked with the main clock from a $4.608 \mathrm{MHz}$ crystal. An internal clock divider can be used to lower the used clock frequency to $2.304 \mathrm{MHz}$ and thereby the power consumption for the microcontroller can be reduced to almost in half, while maintaining functionality. If the microcontroller has a context dependent load it is possible to dynamically adapt the operating frequency as the context of the system changes.

The power consumption reduction can be seen in the measurements in table 2 below. The reason that the reduction is not quite $50 \%$ is that not all parts of the microcontroller are clocked by the main clock and hence their power consumption is not dependent of the frequency.

Table 2. Power consumption depending on operating frequency

\begin{tabular}{|c|c|}
\hline $4.608 \mathrm{MHz}$ & $2.304 \mathrm{MHz}$ \\
\hline $38 \mathrm{~mW}$ & $21 \mathrm{~mW}$ \\
\hline
\end{tabular}

\section{CRC Calculations}

A significant part of the calculations involved in sending PPP packets over RFCOMM is to calculate the CRC checksums. Analyzing the examined system with a profiling tool shows that the CRC calculation is one of the most time consuming routines. The microcontroller has a dedicated circuit for performing CRC calculations. Using the CRC circuit gives approximately 4 times speedup compared to a CRC calculation in software. The time saved by using the CRC circuit can be used to power down the microcontroller or reduce the frequency and thereby save power.

\section{E. Transport Layer Protocols}

The two main transport layer protocols are TCP and UDP, the most significant difference between the two is that TCP is a reliable protocol meaning that all data sent is guaranteed to reach its destination while UDP uses a "best effort" approach. TCP's higher service level comes at the cost of sending 
ACK's and retransmissions that have a negative effect on the system power consumption.

\section{IV.APPLICATION REQUIREMENTS}

\section{A. The Bluetooth Module}

The power consumption of the Bluetooth module can be reduced by using Sniff mode. However if the sensor application generates heavy traffic, the bandwidth requirements might restrict the possible Sniff interval settings as Sniff mode limits the bandwidth. If the application is dependent on fast response times, a long sleep interval will also have a negative impact on the systems performance. If the bandwidth requirement of the system varies it is beneficial to adapt the interval setting depending on the current bandwidth requirement. In the system used as an example the bandwidth requirement is such that an interval setting higher than 200 timeslots results in packet loss.

\section{B. The Microcontroller}

\section{Wait Mode}

Allowing the microcontroller to sleep when it is idle is a good way to reduce power consumption. In some cases a real time operating system will use hardware timers which cause interrupts that will wake up the microcontroller; in such cases the gain of using Wait mode is insignificant. In applications that demands quick response times it is not wise to use Wait or Stop modes as they can severely increase the systems response time.

\section{Frequency Reduction}

In applications where the response time is important or where the real time operating system makes Wait mode an inefficient way to reduce power consumption, frequency reduction can be used. When reducing the frequency of the microcontroller it is vital to analyze the system to make sure it is still able to fulfill its real-time constraints.

\section{Transport Layer Protocols}

When choosing protocol for the transport layer it is important to look at the application, if some data loss is acceptable UDP's "best effort" delivery can give some power savings. This power save comes from not having to send ACK's and retransmissions. In application where data loss is not accepted TCP guarantees the delivery of data at the expense of slightly higher power consumption. When connecting the sensor platform locally to a handheld computer using only the Bluetooth link, the Bluetooth protocol stack ensures that no data is lost so there is no need to use the TCP protocol. UDP is particularly beneficial when Bluetooth Sniff mode is used as it save bandwidth so the Sniff interval can be prolonged.

\section{EXPERIMENTAL SETUP}

The experimental setup consists of a SourceMeter from Keitley named 2400 , it was used as voltage source and the current drawn by the device under test was measured. The SourceMeter was controlled via a GPIB interface and the measurements were analyzed in Matlab. The characterization measurements of the Bluetooth module were made with the module isolated on a private printed circuit board [9].

\section{LOW POWER OPTIMIZATIONS AND EXPERIMENTAL RESULTS}

As a final step the proposed power optimizations are tested on the full system equipped with a pulse sensor from Nonin.

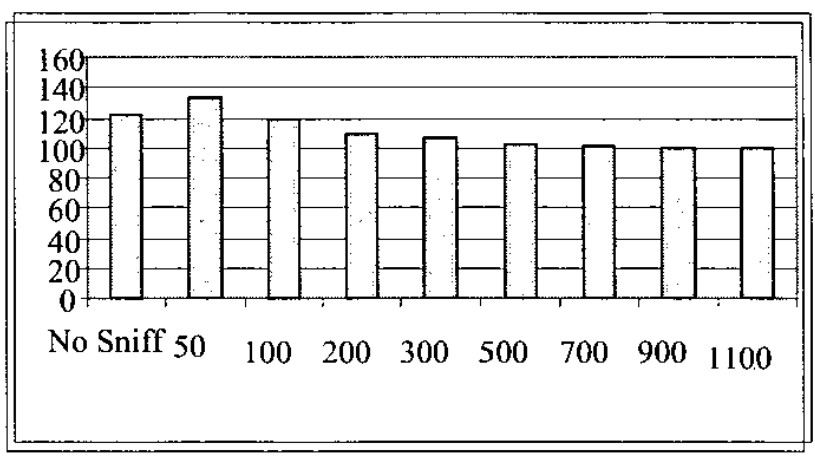

Fig. 4. Power Consumption (mW) of the sensor system depending on Bluetooth Sniff interval

The final tests presented in Figure 4 shows a good correlation with the characterization results in Figure 3. However the improvements are not as clearly seen in the final measurement, as the measurement is done on the whole system including a pulse sensor.

Table 3. Power consumption of the Sensor system depending of Wait mode usage on the Microcontroller.

\begin{tabular}{|c|c|c}
\hline & Wait Mode & No Wait Mode \\
\hline Sending Data & $122 \mathrm{~mW}$ \\
\hline Idle & $122 \mathrm{~mW}$ & $113 \mathrm{~mW}$ \\
\hline
\end{tabular}

The sensor application was written to maximize performance when sending data. To achieve this Wait mode could not be used, so no reduction in power consumption could be seen while the application was sending data. However in situations with lower performance requirements Wait mode can be efficiently utilized 


\section{CONCLUSION}

Several conclusions emerge from the presented design methodology.

Our experiments show that by using the Bluetooth power save modes the power consumption of the module can be reduced by up to $50 \%$. By controlling the power save modes adaptively this can be achieved with sustained performance.

As shown by our measurements the power consumption of the microcontroller approximately scales linearly to the clock frequency. By dynamically controlling the clock frequency depending on the current load situation a good power/performance trade-off can be reached.

A real time operating system can be an impediment when making power optimizations as it does not give the system designer full control over the hardware.

The presented design methodology which is verified by experimental results is a promising first step in prolonging operating time of battery powered wireless embedded Internet systems.

\section{VIIl. FUTURE WORK}

To further improve the characterization of the Bluetooth module it is suggested that the available bandwidth for each Sniff interval is examined.

In order to take full advantage of the Wait modes in the microcontroller, it is necessary to make changes to the sensor application.

To reduce the required bandwidth IP header compression could be implemented.

The dynamic power consumption scale exponentially with the supply voltage according to equation ( 1 ), hence it is possible to reduce the power consumption of the microcontroller significantly by applying an adaptive approach that reduces the supply voltage when the frequency is lowered.

By moving some of the Bluetooth protocols from the microcontroller to the Bluetooth module, it is expected that the power consumption will be reduced since the built-in microcontroller-core in the Bluetooth module is using a lower supply voltage than the microcontroller.

\section{REFERENCES}

[1] A. Östmark, L. Svensson. P. Lindgren, J. Delsing, "Mobile Medical Applications Made Feasible Through Usc of EIS Platforms" in IEEE Instramentation and Measurement Technology Conference, 2003, pp. 292-295.

[2] T. Könberg, C. Öhult, J. Delsing, "Measuring Breathing- and Heart Rate Data with Distribution over Wireless IP Networks" in IEEE Instrzmentation and Measurement Technology Conference, 2003, pp. 888-891.

[3] MI6C/62M Group (Low voltage version) Data Sheet REV.BI,

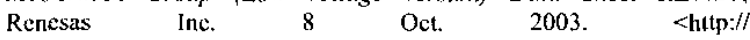
www.renesas.com/avs/resource/japan/eng/pdf/mpumcu/62meds.pdf $>$

[4] Bluetooth Module WML-C10 Class 2. Mitsumi. 8 Oct. 2003. <http:// www.mitsumi.co.jp/Catalog/hifreq/commun/wml/c10/text0le.pdf $>$
[5] Specification of the Bluetooth System, Version 1.1 (200I). $10 \mathrm{Fcb}$. 2003 http://www.bluetooth.org

[6] lwBT - A Lightweight Bluetooth Stack. EISLAB, Luleå University of Technology. 10 Oct. $2003<$ hitp://www.sm.luth.se/ conny/lwbt/>

[7] JwIP - A Lightweight TCP/IP Stack. SICS. 10 Oct. 2003 $<$ http://www.sics.se/ adam/lwip/>

[8] Concept of the power control, Renesas, 13 Feb, $2003<$ http:// www.renesas.com/avs/resource/japan/eng/pdf/mpumcu/apmcl 02e.pdf $>$

[9] J. Eliasson, "Design and evaluation of a Bluctooth enabled system based on a MicroBlaze soft processor", Master's thesis, Lulea University of Tcchnology, Jun 2003. ISSN 1402-16I7; NR 2003:194 